\title{
Lasers for coherent optical satellite links with large dynamics
}

\author{
Nicola Chiodo, Khelifa Djerroud, Ouali Acef, André Clairon, and Peter Wolf* \\ LNE-SYRTE, Observatoire de Paris, CNRS, LNE, UPMC, 61 av. de l'Observatoire, \\ 75014 Paris, France \\ *Corresponding author: Peter.Wolf@obspm.fr
}

\begin{abstract}
We present the experimental realization of a laser system for ground to satellite optical Doppler ranging at the atmospheric turbulence limit. Such a system needs to display good frequency stability (a few parts in $10^{-14}$ ) whilst allowing large and well controlled frequency sweeps of $\pm 12 \mathrm{GHz}$ at rates exceeding $100 \mathrm{MHz} / \mathrm{s}$. Furthermore it needs to be sufficiently compact and robust for transportation to different astronomical observation sites where it is to be interfaced with satellite ranging telescopes. We demonstrate that our system fulfills those requirements and should therefore allow operation of ground to low Earth orbit satellite coherent optical links limited only by atmospheric turbulence. (c) 2018 Optical Society of America

OCIS codes: (140.3425) Laser stabilization; (140.3600) Lasers, tunable; (120.3940) Metrology; (120.3930) Metrological instrumentation; (060.2605) Free-space optical communication; (010.1330) Atmo-
\end{abstract} spheric turbulence

\section{Introduction}

An increasing number of applications require laser sources with low frequency noise, large frequency dynamics, or both. Examples are LIDAR, optical communications to satellites, optical Doppler ranging and telemetry to satellites or between satellites, optical spectrum analyzers, coherent manipulation of atoms for quantum processing, and low noise interferometric sensors.

In this work we consider one particular application: ground to satellite optical Doppler ranging at the atmospheric turbulence limit. That technique uses phase coherent tracking of an optical signal reflected from a corner cube onboard a low Earth orbit satellite, thereby measuring the optical distance variations between the ground telescope and the satellite with a potential for several orders of magnitude improvement over standard satellite laser ranging techniques. More importantly, such measurements, when limited by atmospheric turbulence, provide valuable information on the phase and amplitude noise of that effect (up and down link) of particular importance for phase coherent optical communications [1] and long distance optical free space clock comparisons [2,3. The latter is of particular interest in the context of next generation atomic clocks for space applications in fundamental physics, navigation, and geodesy.

In 2009 coherent optical Doppler ranging to a stationary ground target has demonstrated the feasibility of the method and its limitations from atmospheric turbulence on a $5 \mathrm{~km}$ horizontal link [2]. Furthermore, that experiment has allowed the estimation of the residual noise from atmospheric turbulence in long distance clock comparisons, with very promising results. Those estimations have been confirmed experimentally in another $2 \mathrm{~km}$ horizontal ground link very recently $[\underline{3}$. However similar experiments for ground-space links are still missing and direly needed in order to demonstrate the feasibility under much more difficult conditions (large Doppler dynamics, low return power, high frequency stability requirements of the source). Of interest 
in their own right for precision satellite ranging they are also crucial for the characterization of atmospheric turbulence phase and amplitude noise.

Here we report on progress towards the realization of that goal. We describe the experimental development of the required lasers that need to display state of the art frequency stability, whilst being capable of a controlled frequency sweep spanning $>20 \mathrm{GHz}$ at a maximum rate exceeding $100 \mathrm{MHz} / \mathrm{s}$ in order to compensate the Doppler effect due to satellite motion. We derive the requirements on the laser stability from the expected turbulence noise and show that our laser system meets those requirements.

The application described above requires high stability and controlled and broad frequency tunability at the same time, which is a challenge to many laser stabilization schemes (eg. Fabry-Perot cavities or locking to atomic/molecular transitions). A solution is to use large arm length unbalance fibre interferometers 4]. It has been demonstrated that such systems display state of the art stability with simultaneous sweep linearity [5]. However, the achieved sweep rates (up to $40 \mathrm{MHz} / \mathrm{s}$ ) and range $(<1 \mathrm{GHz}$ ) are insufficient for our purposes. A system with much faster and broader tuning was reported in [6, 7, however with insufficient stability for our purposes.

Our own system is comprised of two lasers each stabilized on an unequal arm fibre interferometer closely inspired by [5]. The system is less stable than the one reported in [5] but still sufficient for our purposes, whilst having broader and faster tuning capability. It is a compromise between compactness and performance as it has to be moved frequently to astronomical sites where it will be used to realize first coherent groundsatellite optical Doppler ranging. It has recently been transported from Paris to the Observatoire de la côte d'Azur in southern France where first preliminary tests with the $1.5 \mathrm{~m}$ lunar/satellite ranging telescope have taken place. Further tests are planned in 2014.

The paper is organized as follows. We first describe the application ie. the planned experiment for a coherent optical ground to satellite link, and derive the resulting requirements on the lasers. We then provide the details of our laser system designed and built for that purpose. This is followed by the experimental results of the laser frequency stability in different configurations (no sweep, linear sweep, non-linear sweep). Finally we discuss those results and conclude.

\section{Requirements for the lasers}

The laser system described here was developed within the scope of the Mini-DOLL (Miniature Deep-space Optical Laser Link) project that aims at demonstrating the feasibility and studying the phase noise limitations of coherent ground to satellite optical links across large distances as proposed for distant clock comparisons and communication across the solar system [8, 9. Mini-DOLL uses a two way link with the light reflected off a corner cube onboard low Earth orbit satellites (around $1000 \mathrm{~km}$ altitude). Thus the signal crosses the atmosphere twice and is subject to the large Doppler dynamics of low Earth orbit satellites. Concerning the received power, we expect less than $1 \mathrm{pW}$ power in the return signal due mainly to the two-way configuration and the small size of the corner cubes $(\approx 3 \mathrm{~cm})$. This is only about an order of magnitude more than the received power from $1 \mathrm{~W}$ emission from a spacecraft at 3 astronomical units from the Earth [9] ie. if successful Mini-DOLL demonstrates the feasibility of such a deep space link with similar received power levels but significantly more adverse Doppler conditions and a double (rather than single) crossing of the atmosphere.

Consider a ground to satellite link as shown in fig. 1 At the instant $t_{1}$ the laser is emitted towards the satellite where, at time $t_{2}$, it is reflected by the corner cube and received back at the ground telescope at instant $t_{3}$. The measurement is the beatnote (fractional frequency difference $y_{b}\left(t_{3}\right)$ ) between the local laser and the signal returning from the satellite. 
The measured beatnote can be written as

$$
y_{b}\left(t_{3}\right)=y_{l}\left(t_{3}\right)-y_{l}\left(t_{1}\right)+2 \frac{v_{s}}{c}\left(t_{2}\right)-\frac{v_{g}}{c}\left(t_{1}\right)-\frac{v_{g}}{c}\left(t_{3}\right)+y_{a t m}\left(t_{1}\right)+y_{a t m}\left(t_{3}\right)
$$

where $y_{l}(t)$ is the laser frequency at instant $t$ (normalized to the nominal laser frequency), $v_{s}(t)$ and $v_{g}(t)$ are the satellite and ground station velocities respectively projected onto the line of sight, $y_{a t m}(t)$ is the perturbation in fractional frequency due to the crossing of the atmosphere (turbulence), and $c$ is the velocity of light.

The aim is to determine the satellite motion (velocity terms in (11)) limited only by atmospheric turbulence (yatm terms in (1)). For the laser stability this implies

$$
y_{l}\left(t_{3}\right)-y_{l}\left(t_{1}\right) \leq y_{a t m}\left(t_{1}\right)+y_{a t m}\left(t_{3}\right)
$$

which in terms of power spectral densities (PSD) becomed 1

$$
\left(1-\cos \left(2 \pi f T_{13}\right)\right) S_{\text {laser }}(f) \leq\left(1+\cos \left(2 \pi f T_{13}\right)\right) S_{a t m}(f)
$$

where $T_{13}=t_{3}-t_{1}$, and $S_{\text {laser }}(f), S_{a t m}(f)$ are the noise PSD of the laser and the atmospheric perturbation respectively.

Equation (3) defines our requirements on laser stability, from an assumption on the noise induced by atmospheric turbulence and from a knowledge of the ground to satellite distance ie. $T_{13}$. Throughout the paper we will assume that the atmospheric noise PSD is of the order of the one measured in [2, 3 , as justified in [2], and that the satellites that we aim at are in low Earth orbit typically at altitudes around $1000 \mathrm{~km}$ giving $T_{13} \approx 6.6 \mathrm{~ms}$.

Other requirements for our experiment come from the expected low signal power and the large Doppler effect induced by satellite motion. We expect that the signal power will be attenuated by at least a factor $10^{-13}$, due to divergence of the beam, small size of the corner cube, attenuation of the atmosphere, losses in the telescope and detection optics, and other effects. Emitting at $\approx 30 \mathrm{~W}$ this implies a return power of the order of $1 \mathrm{pW}$. To achieve a reasonable, shot noise limited, signal to noise ratio the signal needs to be filtered in a narrow band of about $1 \mathrm{kHz}$. The expected $\mathrm{S} / \mathrm{N}$ ratio is then of the order $30 \mathrm{~dB}$ at our 1064 nm wavelength, and expected to fluctuate due to atmospheric scintillation. However, a typical satellite at $1000 \mathrm{~km}$ altitude will induce Doppler shifts varying from $+12 \mathrm{GHz}$ to $-12 \mathrm{GHz}$ during the satellite pass at a maximum rate of about $120 \mathrm{MHz} / \mathrm{s}$. It is not possible to measure a beatnote in a $1 \mathrm{kHz}$ band that varies between 0 and $12 \mathrm{GHz}$ at that rate, so we use the following approach to overcome the Doppler effect. Our experiment is composed of 2 lasers, a local oscillator and an emitting laser. The emitting laser will be at constant frequency while the laser frequency of the local oscillator will be swept to compensate the Doppler shift of the returning signal. We will sweep the frequency of the local oscillator according to the satellite orbit prediction. The result is a beatnote that varies sufficiently slowly to be tracked with a $1 \mathrm{kHz}$ bandwidth (ie. it stays within a $1 \mathrm{kHz}$ band for at least a few $\mathrm{ms}$ ).

In conclusion, the requirements on our laser system can be summarized as follows:

- Stabilize the emitting laser and local oscillator laser to a level satisfying (3).

- Allow for a controlled sweep of the local oscillator laser to compensate for the predicted (theoretical) Doppler shift due to satellite motion $(+12 \mathrm{GHz}$ to $-12 \mathrm{GHz}$ at a maximum rate of $120 \mathrm{MHz} / \mathrm{s})$.

- Ensure that the difference between the actual laser frequency during the sweep and the predicted sweep has a stability satisfying (3).

\footnotetext{
${ }^{1}$ The PSD of $x(t)=n(t) \pm n(t-T)$, where $n(t)$ is a stochastic process, is given by $S_{x}(f)=(1 \pm \cos (2 \pi f T)) S_{n}(f)$
} 
- Allow for large emission power (> $10 \mathrm{~W})$ without deteriorating the frequency stability.

\section{Laser setup}

In our setup we use two lasers one for emission (Nd:YAG laser + Er:Yb multi-stage fibre amplifier) and a broadly tunable Fibre Bragg Grating (FBG) laser as the local oscillator. The set-up is shown schematically in figure 2

The stabilization and sweep control are based on the method described in [5], and the reader is referred to that article for details. In our case we stabilize our two lasers on a $1 \mathrm{~km}$ (local oscillator) and $2 \mathrm{~km}$ (emitting laser) single mode fibre delay line, which are both housed in the same temperature controlled and seismically isolated box. The detailed scheme is shown in figure 3 .

Due to the arm imbalance $\tau(\tau \approx 10 \mu \mathrm{s}$ and $20 \mu$ s for the $1 \mathrm{~km}$ and $2 \mathrm{~km}$ spools respectively) of the Michelson-Morley interferometers, the beatnote frequency on photodiodes PD1 and PD2 depends on $\tau \dot{\nu}_{\text {laser }}(t)$ ie. on the laser frequency derivative. Therefore, by adding a frequency $\Delta \nu_{r f}(t)$ to the demodulation signal of the fibre laser, in closed loop operation (error signal $=0$ ), its frequency is varied (swept) according to the condition

$$
\dot{\nu}_{\text {laser }}(t)=\frac{\Delta \nu_{r f}(t)}{\tau} .
$$

The programmed rf frequency for the sweep $\Delta \nu_{r f}$ is provided by a direct digital synthesizer (DDS), to which we upload the predicted satellite Doppler shifts before the pass. During the satellite pass (about 20 $\min )$ the required laser frequency derivative $\dot{\nu}_{\text {laser }}$ varies from 0 to $120 \mathrm{MHz} / \mathrm{s}$ and back which requires generating an appropriate ramp of $\Delta \nu_{r f}$ varying from 0 to $1.2 \mathrm{kHz}$ according to equ. (44). The DDS generates a new frequency every millisecond according to a previously uploaded file. Additionally it allows for manual frequency steps or time steps during the pre-programmed sequence in order to be able to correct "in real time" for orbit prediction errors. Any manual adjustments (their value and the time at which they are applied) are logged in a file for later traceability.

The emitting laser is a Nd:YAG oscillator emitting at $1064 \mathrm{~nm}$ (manufactured by Innolight). The maximum output power is $500 \mathrm{~mW}$, the spectral linewidth (over $100 \mathrm{~ms}$ ) is $1 \mathrm{kHz}$. The wavelength can be tuned by acting on the temperature by $30 \mathrm{GHz}$, and by acting on the piezo-electric actuator (PZT), mounted on one mirror of the laser cavity, by $\pm 100 \mathrm{MHz}$. The fibre amplifier is manufactured by Nufern and provides 50 W maximum output. The local oscillator is a DFB Yb:fiber laser emitting at $1064 \mathrm{~nm}$ (manufactured by NKT photonics). The maximum output power is $100 \mathrm{~mW}$, the spectral linewidth inferior to $10 \mathrm{kHz}$. The wavelength can be tuned by acting on the temperature by $\sim 120 \mathrm{GHz}$, and by acting on the PZT by 106 $\mathrm{GHz}$ (wide piezo tuning option of NKT-photonics). To sweep the laser for Doppler compensation, we have chosen to act on the PZT of the local oscillator only. The typical optical power sent into each interferometer is $\approx 50 \mu \mathrm{W}$.

Different devices and techniques are used to ensure the thermal, vibration and pressure isolation of the fiber spools. All these effects worsen the performances of the stabilization system in different ways. For thermal and pressure isolation we use 4 boxes one inside the other. The 2 fiber spools are inserted in a metallic box with diameter $30 \mathrm{~cm}$ and height $20 \mathrm{~cm}$. This box is inserted in another metallic box with cylindrical shape.

Inside this second box, but not in thermal contact with the box of the fibers, are placed the 3 AOMs (Acousto Optic Modulators) of the interferometers (AOM 2, 3, and 5 in fig. (3). The second box is actively thermally stabilized by 4 peltier modules attached on the external lateral surface. On the lid of the second box are placed the 2 photodiodes.

The 2 metallic boxes are air-sealed to attenuate pressure variations. The second box is placed inside another metallic box of cubic shape. The inside of the cubic box is covered with $5 \mathrm{~cm}$ thin acoustic absorber 
(polyurethane agglomerate) to attenuate acoustic vibrations. The cubic box is mounted on an active vibration table that filters the seismic vibrations between 10 and $100 \mathrm{~Hz}$.

To dissipate the heat of the peltier elements, they are in thermal contact with the ground by means of 4 twisted copper wires. Finally the cubic box is housed inside a wooden cubic box to reduce fluctuations of room temperature, to further attenuate acoustic noise and to protect the complete set-up. The inside of the wooden box is covered with $5 \mathrm{~cm}$ thick acoustic absorber (polyurethane agglomerate) to attenuate acoustic vibrations. From the outside the complete stabilization set-up is then a wooden cube of $86 \mathrm{~cm}$ sides.

The stabilized temperature of the fiber spool has been studied. We measure the temperature inside the box containing the fiber spool, the floor temperature and the room temperature. The experimental setup is located in an air conditioned laboratory at $22 \pm 1^{\circ} \mathrm{C}$.

Figure 4 shows that, as expected, the temperature of the box of the fiber spool is strongly correlated on the long term with the temperature of the floor, that is used for dissipating the heat inside the stabilization boxes. The temperature drift for the floor temperature is $42.1 \mathrm{mK} /$ day, while for the inner box is $1.5 \mathrm{mK} /$ day: the protection system attenuates the long term temperature variations by a factor 27 .

However, the diurnal variations of the environment temperature also affect the temperature of the fiber spool. By analyzing the residual of the temperatures after removal of the linear drift (fig [5), we can see that the room and box temperatures have correlated diurnal variations, with the box being delayed by about 15 hours, and attenuated by about a factor 200. Practically no diurnal variations are visible on the floor temperature residuals.

We want to verify that the linear drift of the temperature of the inner box corresponds to the linear frequency drift of the stabilized Nd:YAG laser (a few hundred $\mathrm{Hz} / \mathrm{s}$ ) when measured with respect to an independent outside reference (see section 4 ). The relation between a temperature change $(\Delta \theta)$ and a frequency change due to an optical length variation of the fibre is

$$
\frac{\Delta \nu_{\text {laser }}}{\nu_{\text {laser }}}=\frac{2 \Delta l}{l}=2 S \Delta \theta
$$

where $S$ is the coefficient of optical length expansion of our fibres $S \sim 8 \times 10^{-6} \mathrm{~K}^{-1}$ [10. The measured long term temperature drift over several days is $\sim 1.7 \times 10^{-8} \mathrm{~K} / \mathrm{s}$ (see above) giving a frequency drift of only $\sim 80 \mathrm{~Hz} / \mathrm{s}$, somewhat less than observed. However, the frequency measurements were carried out over much shorter intervals, typically a few minutes. As the inset of figure 4 shows, the temperature drift over such short periods can reach up to $8 \times 10^{-7} \mathrm{~K} / \mathrm{s}$ which is compatible with the observed frequency drifts of a few hundred $\mathrm{Hz} / \mathrm{s}$.

\section{Frequency stability of the lasers}

The lasers are characterized in terms of power spectral density (PSD) and Allan deviation of the fractional frequency fluctuations of the lasers. To characterize the experimental stability of the lasers, 2 different measurements have been performed.

The first one is the comparison between the 2 lasers of the experiment, the local oscillator and the emitting laser. We aquire the beat-note between the 2 stabilized lasers with a fast frequency counter (Brilliant Instruments BI200). The counter can measure from DC up to $2 \mathrm{GHz}$. The counter noise of the instrument depends on the measured frequency, but is negligible in all our measurements for Fourier frequencies below $100 \mathrm{kHz}$. The 2 lasers are combined in a fiber coupler, and the output is focused on a free space fast photodiode. All the RF signals, and the frequency counter have the same reference, so its frequency noise plays no role in the measurement. Three measurements at different sampling times are performed: at $1 \mu \mathrm{s}$, at $10 \mu \mathrm{s}$ and at $1 \mathrm{~ms}$. The linear drift between the 2 stabilized lasers is of the order of a few $\mathrm{Hz} / \mathrm{s}$ over the measurement intervals (typically a few minutes). 
The noise of the 2 stabilized lasers is correlated because the stabilization system (spools, AOMs, diodes, etc..) are located in the same box. As a consequence the absolute phase noise of the lasers is certainly higher than when measured relative to each other.

Therefore, the stabilized emitting laser has also been compared with a completely independent system (a cryogenic sapphire oscillator at $12 \mathrm{GHz}$ ) using a frequency comb. The laboratories where the lasers of the experiment and the frequency comb are located, are in different buildings and are connected by a $130 \mathrm{~m}$ long optical fiber link. The noise of the return optical fiber link, $260 \mathrm{~m}$ in total, shows an Allan deviation of $1 \times 10^{-14}$ at $1 \mathrm{~s}$ and $5 \times 10^{-15}$ at $10 \mathrm{~s}$, ie. is negligible for our purposes, at least for integration times greater than $\sim 0.1 \mathrm{~s}$.

The sampling time of the frequency comb measurement is $10 \mathrm{~ms}$. The linear drift of the emitting laser with respect to the frequency comb is $600 \mathrm{~Hz} / \mathrm{s}$. Compared to the drift between the 2 stabilized lasers, the drift of the frequency of the emitting laser is about two orders of magnitude worse. This clearly shows that on the long term our two lasers are strongly correlated, as expected.

The performances of the stabilized lasers are shown in fig. 6 and 7. From figure 7 we see that for optimum integration times $(\sim 0.2 \mathrm{~s})$ the level of the noise of the stabilized laser is $\sim 5$ times worse when compared to an independent source than when the two lasers are compared between them. For comparison we have also plotted in figure [6 the expected noise that will limit our satellite experiment, ie. the expected atmospheric turbulence noise multiplied by the transfer function from equ. (3) $\left(S_{a t m}(f)\left(1+\cos \left(2 \pi f T_{13}\right)\right) /\left(1-\cos \left(2 \pi f T_{13}\right)\right)\right.$ with $\left.T_{13}=6.66 \mathrm{~ms}\right)$. We note that for Fourier frequencies of interest to us $(<10 \mathrm{~Hz})$ the noise of the lasers will be sufficient for our experiment.

The full experimental setup has been built and tested at the SYRTE, Paris Observatory. In November 2012 it was transported to the Calern site of the Observatoire de la côte d'Azur in southern France at an altitude of $1270 \mathrm{~m}$, in preparation for the satellite tests. In both sites the stabilized lasers remain locked for some hours, but at the Calern site the locking is much more robust maintaining lock for many hours without requiring any adjustments. We have also noted that the typical stability of our two lasers when compared to each other is in general about a factor 2 to 3 better at Calern than in the Paris lab, although the temperature stabilization of the room in Calern (just below the telescope with open cupola etc..) is significantly less good than in the Paris laboratory. Both these effects are most likely due to the lower atmospheric pressure at the Calern altitude leading to less thermal and acoustic coupling between the room and the inside box that contains the fiber spools for the stabilization.

\section{Linear frequency sweep}

Next we have verified that the laser stability is not modified when the local oscillator laser frequency is swept over $20 \mathrm{GHz}$ at different rates. As a first step we carry out purely linear frequency sweeps, which also serve for the precise determination of the delay $\tau$ (c.f. equ. (4) ) required for the controlled non-linear sweep for correct Doppler compensation.

To perform the following measurements, the beat-note between the swept local oscillator and the emitting laser is collected with a $25 \mathrm{GHz}$ fast photodiode. The signal from the photo-detector is divided by 8 by a low noise digital divider that operates from some $\mathrm{kHz}$ to $18 \mathrm{GHz}$ input frequency. The divided signal is then recorded with the frequency counter described in the previous section, that operates up to 2 GHz. The setup allows to measure a frequency change of $32 \mathrm{GHz}$, from $-16 \mathrm{GHz}$ to $+16 \mathrm{GHz}$.

Figure 8 shows a linear frequency sweep at $1 \mathrm{GHz}$ spanning more than $20 \mathrm{GHz}$. The residuals clearly show a quadratic behavior, as expected from the dispersion of the fibre (for our Corning HI1060 fibres the nominal dispersion coefficient around $1060 \mathrm{~nm}$ is $1.43 \times 10^{-22} \mathrm{~s}^{2} / \mathrm{km}$ ). We fit a quadratic model of the local oscillator (LO) frequency to the data, of the form 


$$
\nu_{L O}(t)=\nu_{0}+C t+D t^{2}
$$

obtaining $\mathrm{C}=-1043714018.62 \pm 0.6 \mathrm{~Hz} / \mathrm{s}$ and $\mathrm{D}=-15.470 \pm 0.02 \mathrm{~Hz}^{2} / \mathrm{s}$. Combining equations (4) and (6) we find the relation for the dispersion effect on our delay $d \tau / d \nu=-2 D \tau_{0} / C^{2}$ where $\tau_{0}$ is the delay of our $2 \mathrm{~km}$ (1 km double pass) fibre at the nominal frequency2. We find a dispersion coefficient of $1.36 \times 10^{-22} \mathrm{~s}^{2} / \mathrm{km}$, very close to the value quoted by the manufacturer.

In principle it is possible to take the dispersion effect into account when programming $\Delta \nu_{r f}(t)$ to compensate for the predicted Doppler effect of the satellite. However, in practice this is not necessary as the sweep velocities are low when the frequency is far $(10 \mathrm{GHz})$ from its nominal value (see section 6 and fig. 10). Consequently an error in $\tau$ has a smaller effect at those frequencies ie. when the dispersion effect is largest. For example, at a $10 \mathrm{MHz} / \mathrm{s}$ sweep rate a 3 ps error in tau $(=d \tau / d \nu \times 10 \mathrm{GHz})$ corresponds to a $3 \mathrm{~Hz} / \mathrm{s}$ sweep error, less than the natural drift of our lasers.

Thus we determine a single value of $\tau$ at the central frequency and use it to program $\Delta \nu_{r f}$ for the satellite Doppler correction. To do so the following procedure has been adopted. In the stabilization system of fig. 3 constant values of $\Delta \nu_{r f}$ are introduced in the servo-loop. This corresponds to linearly sweeping the local oscillator. To take into account the natural drift of the laser, for each value of $\Delta \nu_{r f}$, we sweep with the positive value and the negative value. To further take in account the natural drift of the laser, we repeat the same values of $\nu_{r f}$ after some seconds. The set of data of $\Delta \nu_{r f}$ is the following: $+1 \mathrm{kHz},-1 \mathrm{kHz}$, then after 1 second $+2 \mathrm{kHz},-2 \mathrm{kHz},+5 \mathrm{kHz}$ and $-5 \mathrm{kHz}$. After 50 seconds we repeat $+2 \mathrm{kHz}$ and $-2 \mathrm{kHz}$, and after waiting 50 seconds we apply $+5 \mathrm{kHz}$ and $-5 \mathrm{kHz}$. The values of $\Delta \nu_{r f}$ sent to the system, $1 \mathrm{kHz}, 2 \mathrm{kHz}$ and $5 \mathrm{kHz}$, correspond to laser frequency changes of roughly 100, 200 and $500 \mathrm{MHz} / \mathrm{s}$, respectively.

For each $\Delta \nu_{r f}$ value, the laser beatnote is recorded and a linear fit of the data is carried out to compute the values of $\tau$ from which we calculate the average value and its standard deviation. A typical averaged value of $\tau$ is $9.5761890718608 \times 10^{-6} \mathrm{~s}$ with a standard deviation of $2 \times 10^{-12} \mathrm{~s}$. We attribute that uncertainty to the effect of the natural drift of the two lasers during and between measurements. Repeating the measurements, even separated by several days, the obtained value of $\tau$ does not change by more than a few picoseconds.

The instability at different linear sweep velocities has been tested. Fig. 9 shows the PSD of the fractional frequency difference between the 2 lasers, after subtraction of the applied linear sweep. The figure shows that it is possible to linearly sweep the laser up to $1 \mathrm{GHz} / \mathrm{s}$ without significantly degrading the stability. The data comply with the requirement for measuring the turbulence, even taking in account the worsening of the noise level when comparing to an independent laser source (see fig. 6).

Note that the data for fig. 9 were collected months before the data of fig. [6 The new data have been obtained after several improvements of the isolation of the boxes that contain the fiber spools, which resulted in the disappearance of the peaks in the PSD below $20 \mathrm{~Hz}$, probably due to pressure fluctuations of the room.

In summary, we have carried out linear sweeps up to $1 \mathrm{GHz} / \mathrm{s}$ over more than $25 \mathrm{GHz}$ without a noticeable degradation of the laser stability and in compliance with our requirements for the satellite experiment ie. with a noise level below the expected contribution from atmospheric turbulence.

\section{Non-linear frequency sweep}

To evaluate the capability of our experimental setup to compensate the satellite Doppler effect, we have simulated the corresponding frequency shift for a typical satellite pass for our experiment $(800 \mathrm{~km}$ altitude, inclination 98 degrees, pass culminating at 78 degrees elevation), shown in fig. 10. The maximum sweep

\footnotetext{
${ }^{2}$ Substituting the derivative of (6) into (4) with $\tau(\nu)=\tau_{0}+(d \tau / d \nu)\left(\nu-\nu_{0}\right)$ we obtain $C \tau_{0}+\left(2 D \tau_{0}+C^{2}(d \tau / d \nu)\right) t+\mathcal{O}\left(t^{2}, t^{3}\right)=$ $\Delta \nu_{r f}$. Knowing that $\Delta \nu_{r f}$ is constant for all $t$ we have $2 D \tau_{0}+C^{2}(d \tau / d \nu)=0$.
} 
rate is $\sim 120 \mathrm{MHz} / \mathrm{s}$, and it corresponds to a decrease of the frequency of $\sim 24.8 \mathrm{GHz}$. The full duration is 652 seconds.

We measure the beat-note between the two stabilized lasers as in the previous section. After stabilizing the 2 lasers, the beat-note is first increased by $+12 \mathrm{GHz}$ by use of the DDS acting on the PZT of the local oscillator. Next we sweep the local oscillator frequency by introducing the correct data sequence in the DDS. The sampling time for the counter is $1 \mathrm{~ms}$.

As a result, the frequency measured by the counter decreases, then it crosses zero and it restarts to increase up to to $\sim 12 \mathrm{GHz}$. This is due to the fact that the frequency counter measures the absolute value of the frequency difference between the 2 lasers, regardless of the sign. In the measuring setup, there are several components that cut the low frequency resulting in the absence of data from the counter near 0 frequency.

The data from the counter, $\nu_{\text {measured }}$ are processed in the following way. We compute the difference $\nu_{\text {diff }}=\nu_{\text {measured }}-\nu_{\text {prevision }}$ after interpolating the data to ensure that $\nu_{\text {measured }}$ and $\nu_{\text {prevision }}$ are taken at the same instant (see fig 11). The frequency difference $\nu_{\text {diff }}$ represents the error we commit in our Doppler compensation and it includes the natural drift of the beatnote. The remaining linear drift is $5.76 \mathrm{~Hz} / \mathrm{s}$.

In fig. 11, we see the natural relative drift of our lasers but the large signature of the Doppler effect is entirely compensated, ie. the actual frequency sweep corresponds to the intended one to within the natural drift of our lasers. This result also justifies neglecting the dispersion effect (see section 5 ) for our purposes. When computing the PSD and the Allan Deviation of $\nu_{\text {diff }}$ (Fig 12 and 13) we see slightly larger noise than when applying a linear sweep or no sweep, but which is still sufficiently low for our purposes.

\section{Frequency stability of the amplifier}

To maximize the returning signal power we amplify the signal from our stabilized emitting laser using a multiple stage Nufern fibre amplifier (max. $50 \mathrm{~W}$ output). Typically such amplifiers are specified for a minimum seed laser linewidth of about $10 \mathrm{kHz}$, but our stabilized laser has much lower linewidth (around $25 \mathrm{~Hz}$ ) and we require the amplified light to have the same stability characteristics as the seed laser. To ensure that this is the case we have tested a $10 \mathrm{~W}$ model of the same amplifier (on loan from Nufern) before acquiring our $50 \mathrm{~W}$ version. As shown below, the phase noise added by the amplifier is negligible for our purposes, so we use it to amplify our emission laser as the last stage before injection into the telescope. If this had not been the case we could have stabilized the laser+amplifier (extracting a small fraction of light before the telescope) on our fibre spool at the cost of higher complexity. It turns out that this is not necessary.

For the amplifier phase noise measurement we have used a Mach-Zender setup as shown in figure 14. The emitting laser, that is frequency stabilized, is split in 2 parts: one part passes in a fiber pigtailed AOM, which shifts its frequency by $80 \mathrm{MHz}$, the second is amplified and $1 \mathrm{~mW}$ is split off the amplified beam to perform the measurement. The beat-note at $80 \mathrm{MHz}$ is measured as described in sect. 3.

Fig. 15 shows the measurements done with and without amplifier (short-circuiting the amplifier in fig. 14), the latter characterizes the noise floor of our measurements system.

From fig. 15 we conclude that the PSD of the frequency noise added by the amplifier is at or below $10^{-31}$ $\mathrm{Hz}^{-1}$ in fractional frequency $\left(<8 \times 10^{-3} \mathrm{~Hz}^{2} / \mathrm{Hz}\right.$ in absolute frequency) for $10 \mathrm{~Hz}<f<10 \mathrm{kHz}$, and well below the noise of our stabilized emitting laser at all frequencies. The peak at $20 \mathrm{~Hz}$ is most likely due to pressure fluctuations in the laboratory (which we have investigated seperately) and the noise above $\sim 10$ $\mathrm{kHz}$ is consistent with the phase noise of our frequency counter. It seems clear that the observed noise is the noise floor of the measuring setup, so the actual phase noise added by the amplifier is likely to be below that. For comparison, a flat PSD (white frequency noise) at $8 \times 10^{-3} \mathrm{~Hz}^{2} / \mathrm{Hz}$ corresponds to a line-width of $25 \mathrm{mHz}$, ie. we expect that the amplifier does not degrade any seed signal as narrow as that or below, which is remarkable. 


\section{Conclusion}

We have presented an original laser system designed for ground to low Earth orbit satellite coherent optical links. The aim was to develop a system that is capable to cope with the high Doppler dynamics $( \pm 12 \mathrm{GHz}$ with rates up to $120 \mathrm{MHz} / \mathrm{s}$ ) whilst displaying state of the art frequency stability in order to be limited by phase noise from atmospheric turbulence rather than the laser stability. Our solution is based on laser stabilization using unequal arm fibre interferometers closely inspired by [5]. Based on simulated satellite Doppler shifts and expected turbulence conditions taken from [2] we demonstrate that our system satisfies these requirements. A system with better stability was reported in [5], however with sweep rates no higher than $40 \mathrm{MHz} / \mathrm{s}$ and range $<1 \mathrm{GHz}$, that are insufficient for our purposes. Much broader and faster tuning was reported in [6], however with insufficient stability for our purposes. We combine both these features in a compromise between performance and compactness as it has to be moved frequently to astronomical sites where it will be used to realize first coherent ground-satellite optical Doppler ranging.

The system has been transported from Paris to the Observatoire de la côte d'Azur in southern France where it was used with the $1.5 \mathrm{~m}$ lunar/satellite ranging telescope. First tests have encountered difficulties because of the impossibility of using the in situ adaptive optics system as planned. Further tests are planned for 2014 after an upgrade of the adaptive optics system.

\section{Acknowledgements}

Helpful discussions with Giorgio Santarelli and Sébastien Bize are greatfully acknowledged. We would also like to thank Yann Le Coq, Laurent Volodimer, José Pinto and Michel Lours for their valuable contributions. This work was supported by CNES research grant (R-S09/SU-0001-021) DA 10069354, financing by LNE and by the Action Spécifique GRAM (INSU/INP/CNES).

\section{References}

1. M. Gregory et al., Inter-satellite and satellite-ground laser communication links based on homodyne BPSK, Proc. SPIE 7587, 75870E, (2010).

2. K. Djerroud et al., Coherent optical link through the turbulent atmosphere, Opt. Lett. 35, 1479 (2010).

3. F. R. Giorgetta et al., Optical two-way time and frequency transfer over free space, Nature Photonics, Online 28 April (2013), DOI:10.1038/NPHOTON.2013.69. arXiv:1211.4902, (2012).

4. F. Kefelian et al., Ultralow-frequency-noise stabilization of a laser by locking to an optical fiber-delay line, Opt. Lett. 34, 914, (2009).

5. H. Jiang et al., An agile laser with ultra-low frequency noise and high sweep linearity, Opt. Exp. 18, 3284, (2010).

6. P. A. Roos et al., Ultrabroadband optical chirp linearization for precision metrology applications, Opt. Lett. 34, 3692, (2009).

7. Z. W. Barber et al., Characterization of an actively linearized ultrabroadband chirped laser with a fiber-laser optical frequency comb, Opt. Lett. 36, 1152, (2011).

8. P. Wolf et al., Quantum physics exploring gravity in the outer solar system: the SAGAS project, Exp. Astr. 23, 651, (2009).

9. B. Christophe et al., OSS (Outer Solar System): a fundamental and planetary physics mission to Neptune, Triton and the Kuiper Belt, Exp. Astr. 34, 203, (2012).

10. V. Dangui et al., Phase sensitivity to temperature of the fundamental mode in air-guiding photonic bandgap fibers, Opt. Exp. 13, 6669, (2005). 


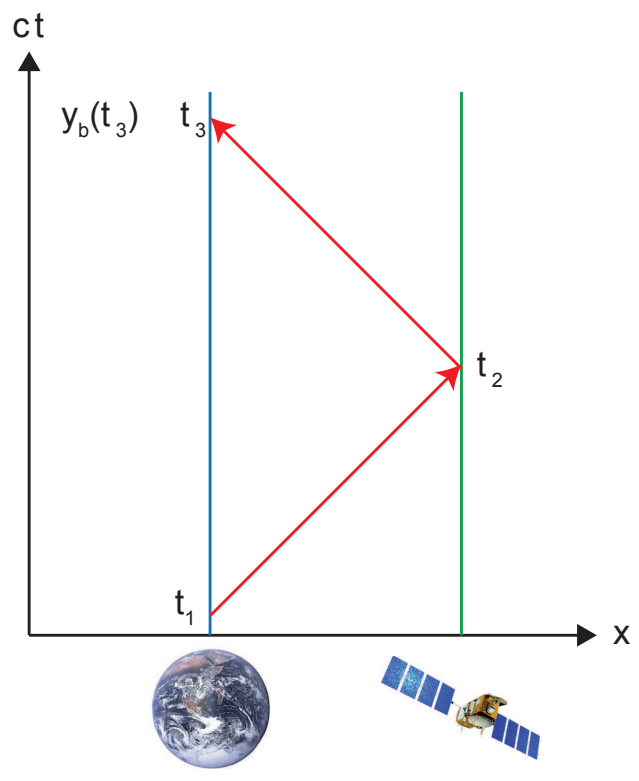

Fig. 1. Space-time diagram of the measurement principle. 


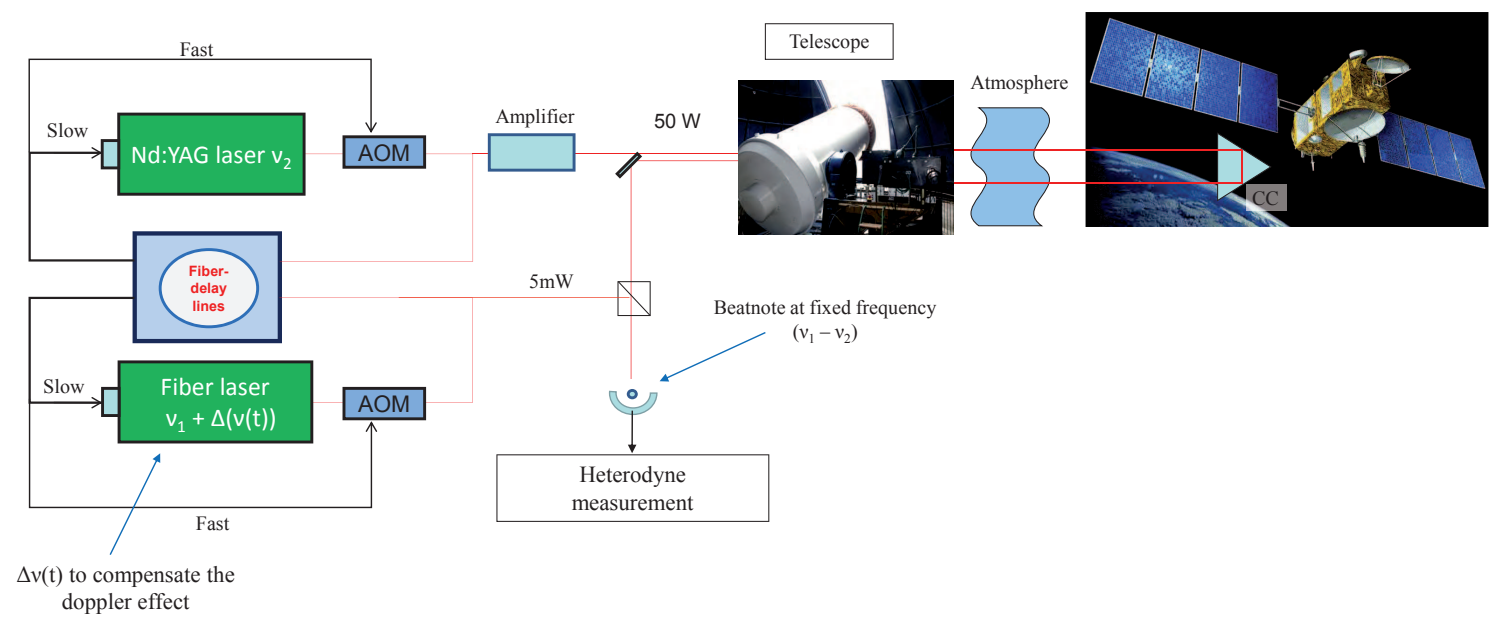

Fig. 2. Laser set-up. The two lasers are stabilized on fibre delay lines. That stabilization is also used to vary the frequency of the local oscillator in order to compensate for the Doppler effect such that the beatnote is at constant frequency. 


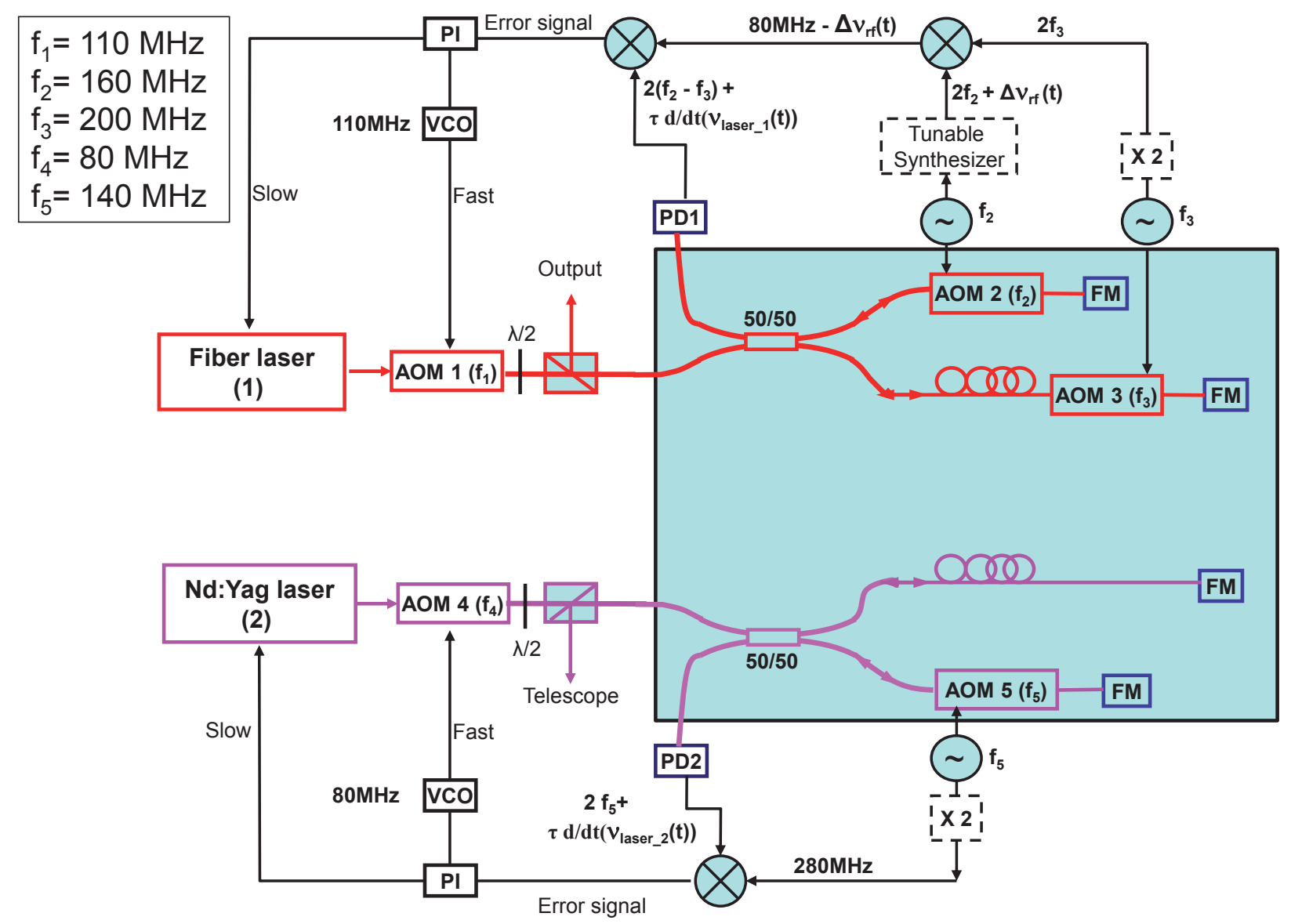

Fig. 3. Laser stabilization scheme. AOM: Acousto-Optic Modulator; FM: Faraday Mirror; PD: Photodiode; VCO: Voltage Controlled Oscillator; PI: Proportional Integrator; $\tau=$ time delay due to arm inbalance. In closed loop operation, the error signals are zero, implying $\dot{\nu}_{\text {laser }}=0$ for the emission laser (YAG laser $\left.(2)\right)$ and $\dot{\nu}_{\text {laser }}(t)=\Delta \nu_{r f}(t) / \tau$ for the local oscillator (Fiber laser (1)). 


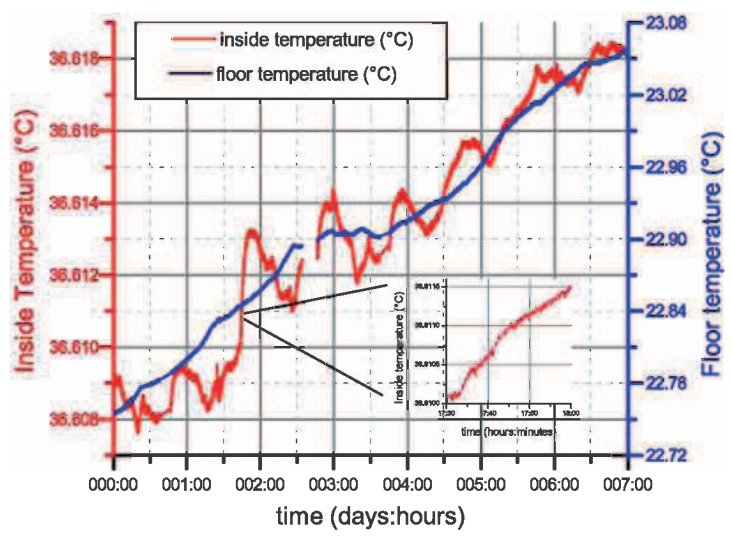

Fig. 4. Temperature over 1 week for the box containing the fiber spools (red) and the floor (blue). 


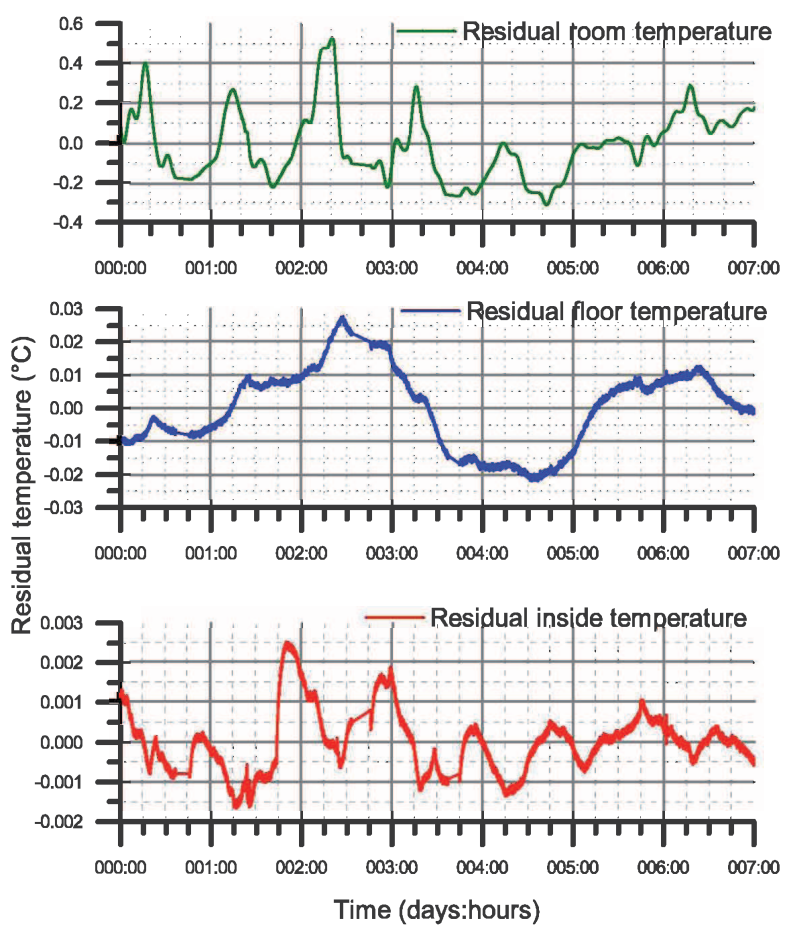

Fig. 5. Residual fluctuations (after linear fit) of the smoothed room temperature (top, olive), floor temperature (middle, blue) and inner temperature (bottom, red). 


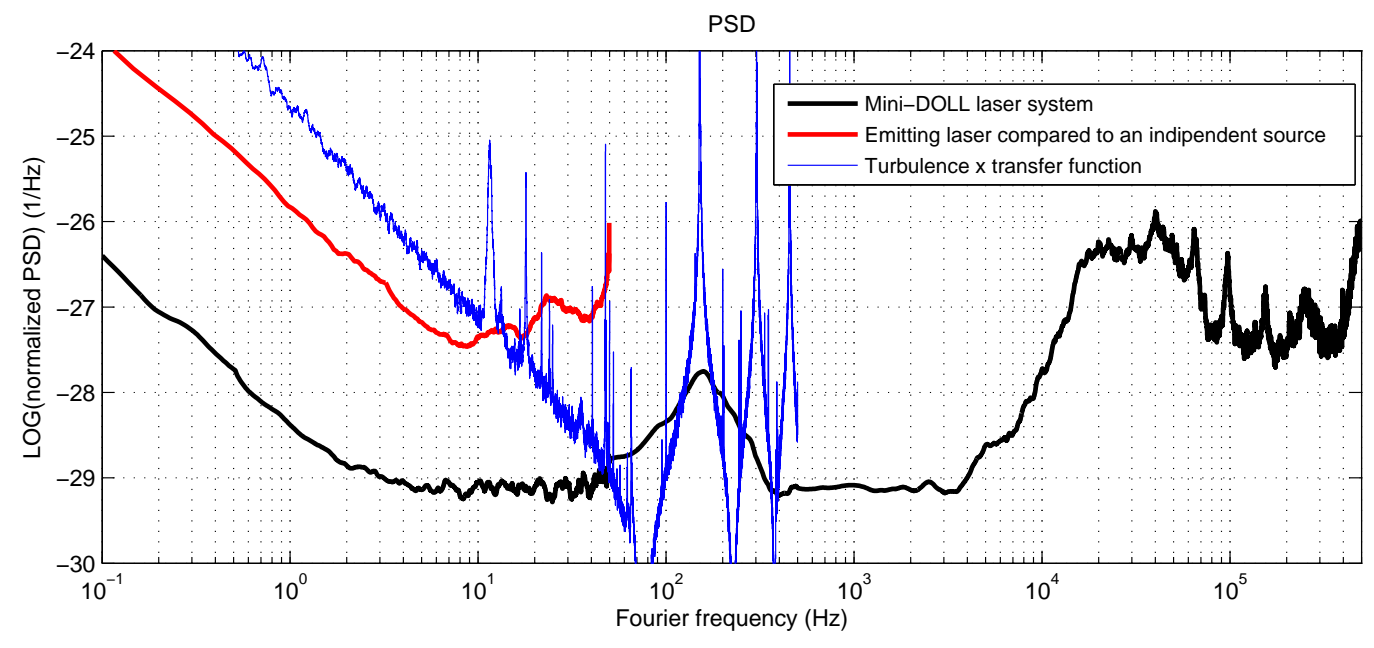

Fig. 6. PSD of fractional frequency $\left(\Delta \nu / \nu_{0}, \nu_{0}=\right.$ laser frequency $\left.=282 \mathrm{THz}\right)$. Black: beatnote between the 2 stabilized lasers. Red: beatnote between the stabilized emitting laser and an independent reference. Blue: expected noise of the turbulence weighted by the transfer function according to (3).

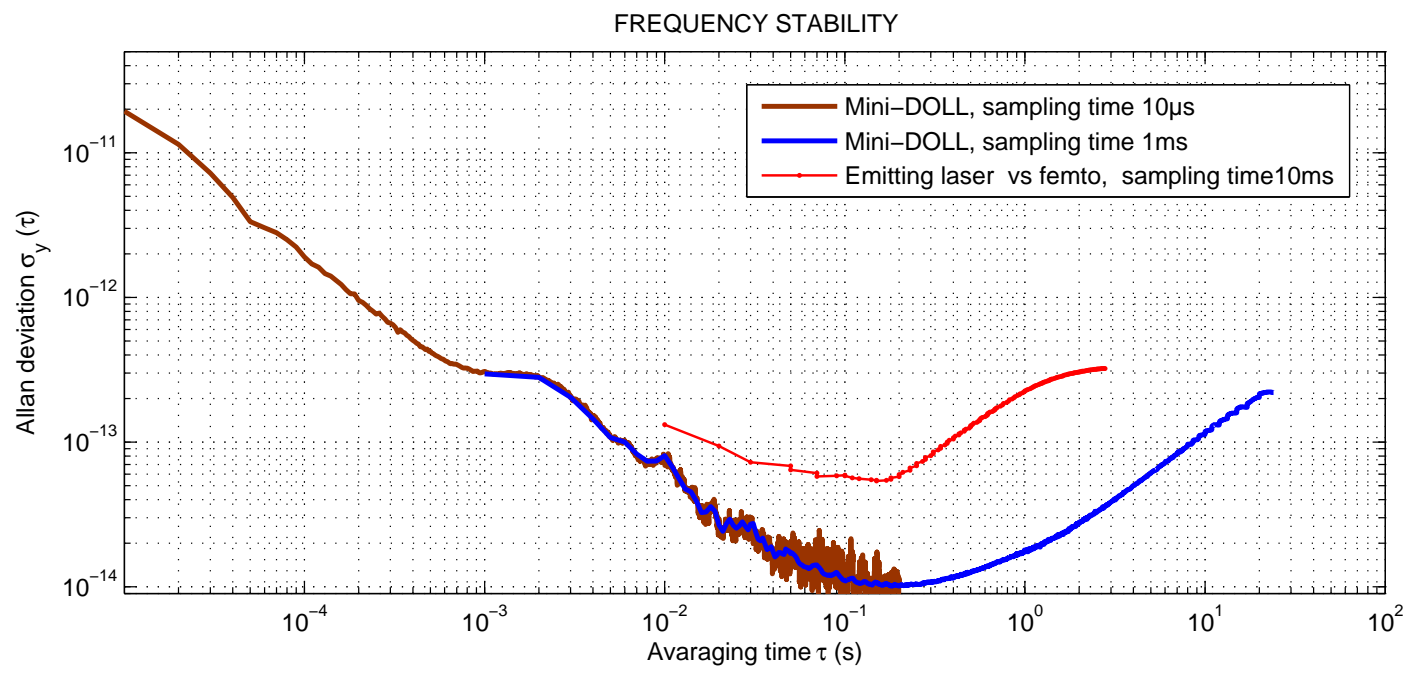

Fig. 7. Allan deviation of the fractional frequency fluctuations between the two stabilized lasers and between the stabilized emitting laser and an independent reference. 

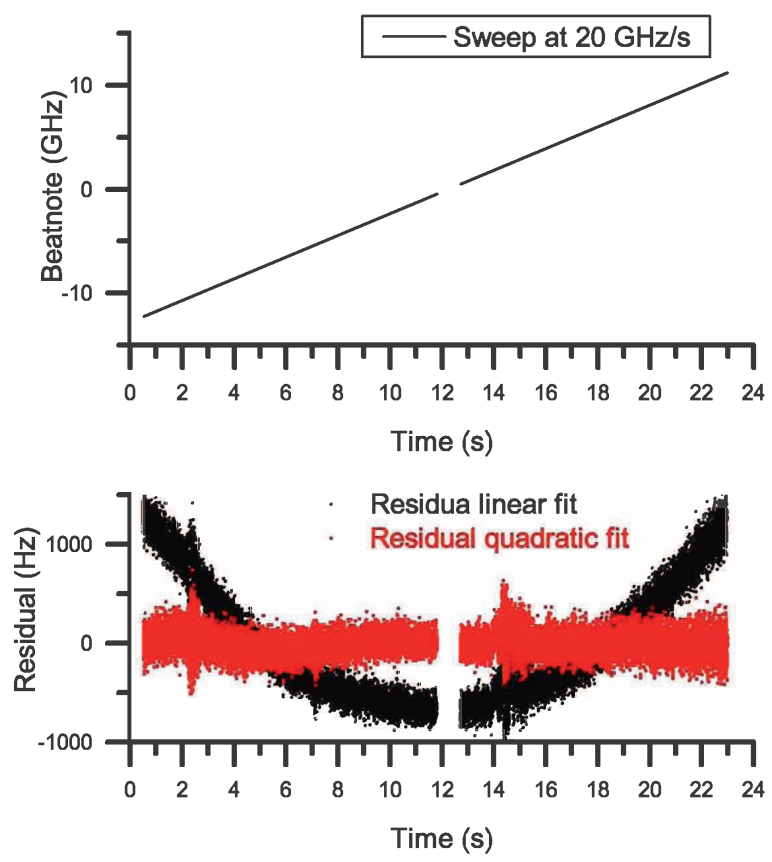

Fig. 8. Top: beatnote $\left(\nu_{\text {emit }}-\nu_{L O}(t)\right)$ between the 2 lasers when the local oscillator (LO) is linearly swept at $1 \mathrm{GHz} / \mathrm{s}$ over more than $20 \mathrm{GHz}$. Bottom: residuals of the beatnote after linear (black) and quadratic (red) fitting. 


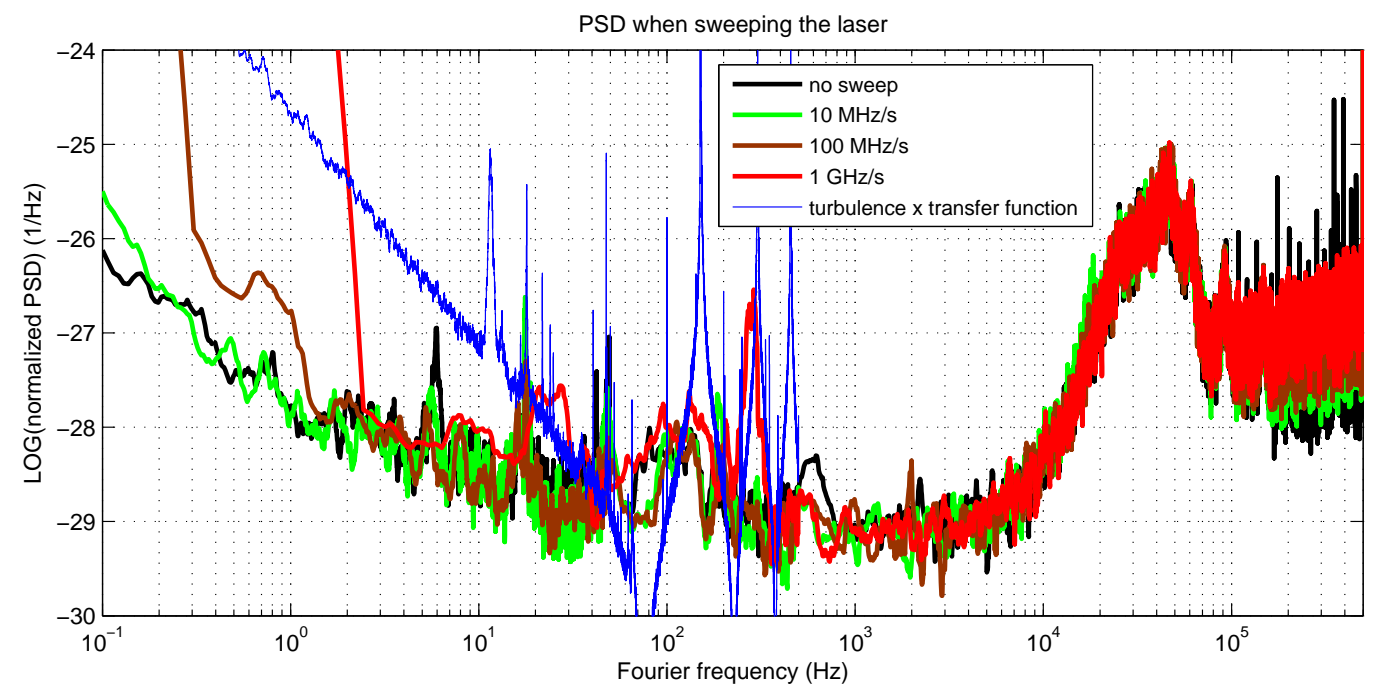

Fig. 9. Fractional frequency PSD of the beatnote between the 2 lasers when the local oscillator is linearly swept up to $1 \mathrm{GHz} / \mathrm{s}$, together with the expected noise of turbulence weighted by the transfer function according to (3).

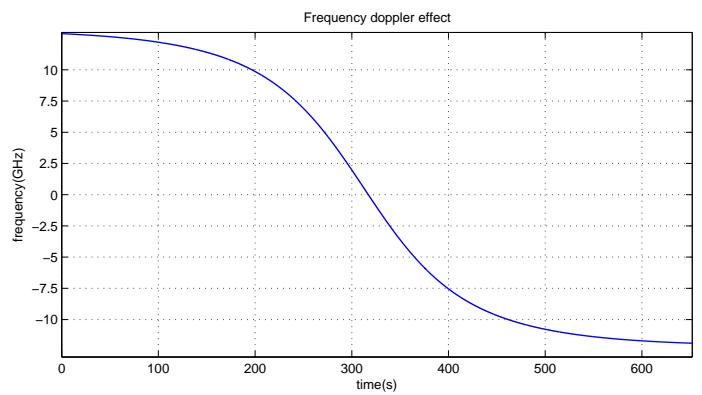

Fig. 10. Simulated Doppler frequency shift for a typical satellite pass 


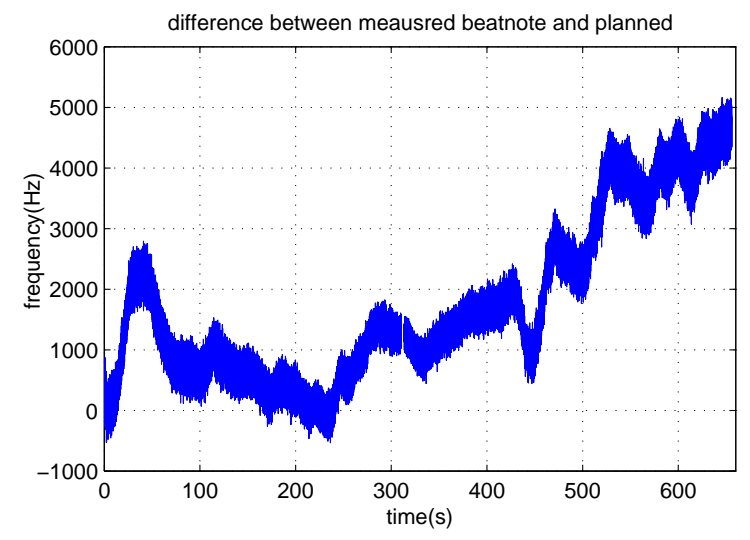

Fig. 11. Difference between the measured beatnote and the frequency Doppler shift simulation, $\nu_{\text {diff }}$.

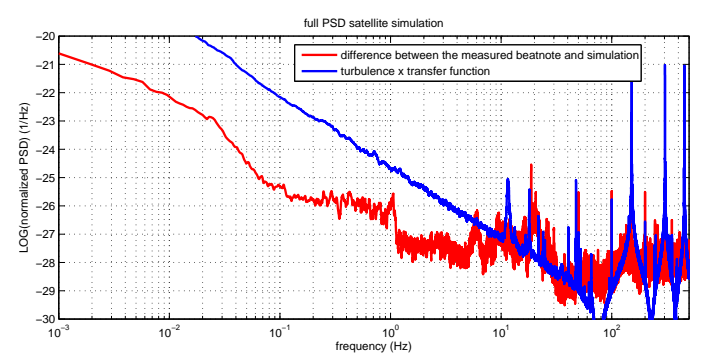

Fig. 12. Fractional frequency PSD of the difference between the measured beatnote and the Doppler shift simulation, $\nu_{\text {diff }}$ (red), together with the expected noise of turbulence weighted by the transfer function according to (3) (blue). 


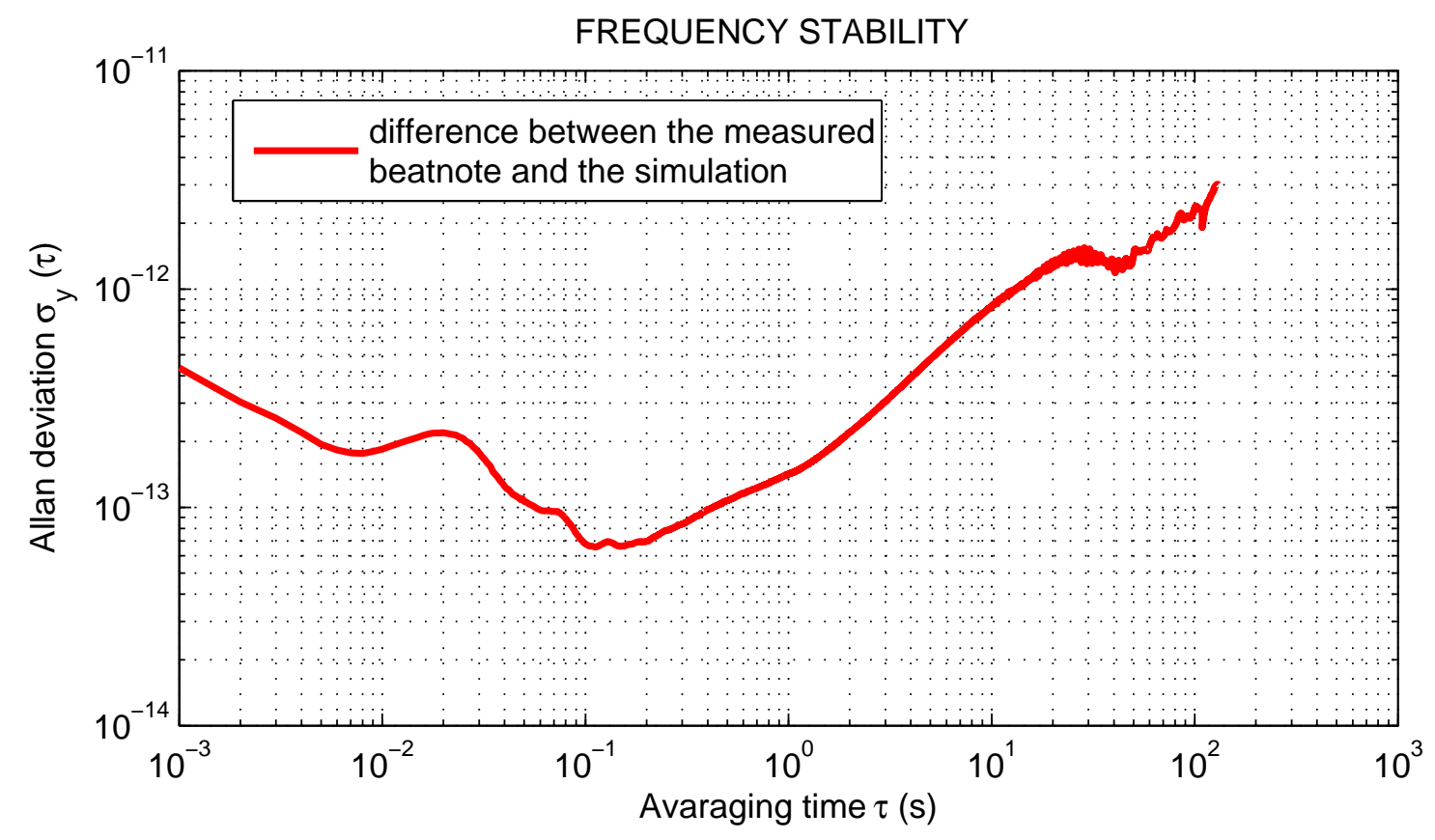

Fig. 13. Allan deviation of the difference between the measured beatnote and the Doppler shift simulation, $\nu_{\text {diff }}$.

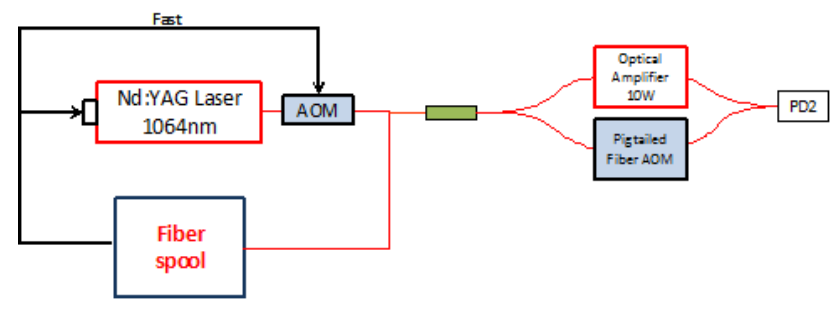

Fig. 14. Experimental setup for measuring the noise of the optical amplifier. 


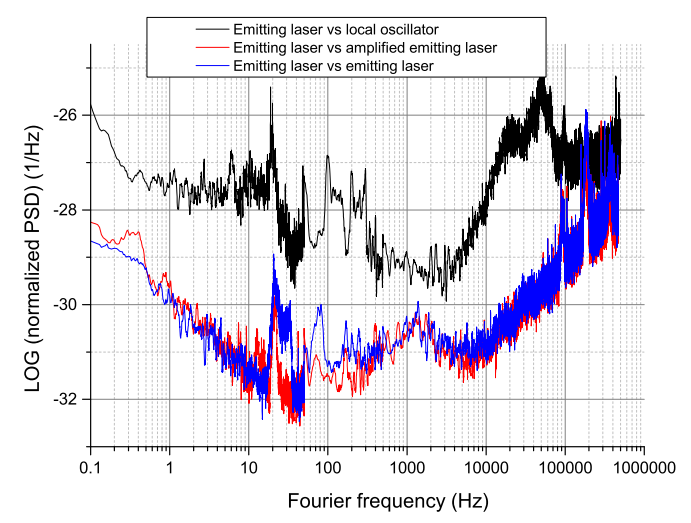

Fig. 15. Fractional frequency PSD. Black: emitting laser compared to local oscillator (for reference). Red: with amplifier. Blue: Without amplifier ("short circuiting" the amplifier in figure 3), the blue line corresponds to the noise floor of our measurement system. 